\title{
Determination of the Strong Coupling Constant from Jet Rates in Deep Inelastic Scattering
}

\author{
H1 Collaboration
}

\begin{abstract}
:
Jet rates in deep inelastic electron proton scattering are studied with the $\mathrm{H} 1$ detector at HERA for momentum transfers squared between 10 and $4000 \mathrm{GeV}^{2}$. It is shown that they can be quantitatively described by perturbative QCD in next to leading order making use of the parton densities of the proton and with the strong coupling constant $\alpha_{s}$ as a free parameter. The measured value, $\alpha_{s}\left(M_{Z}^{2}\right)=$ $0.123 \pm 0.018$, is in agreement both with determinations from $e^{+} e^{-}$annihilation at LEP using the same observable and with the world average.
\end{abstract}




\section{H1 Collaboration}

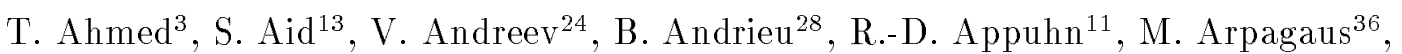

A. Babaev ${ }^{26}$, J. Baehr ${ }^{35}$, J. Bán ${ }^{17}$, P. Baranov ${ }^{24}$, E. Barrelet ${ }^{29}$, W. Bartel ${ }^{11}$, M. Barth ${ }^{4}$,

U. Bassler ${ }^{29}$, H.P. Beck ${ }^{37}$, H.-J. Behrend ${ }^{11}$, A. Belousov ${ }^{24}$, Ch. Berger ${ }^{1}$, H. Bergstein ${ }^{1}$,

G. Bernardi ${ }^{29}$, R. Bernet ${ }^{36}$, G. Bertrand-Coremans ${ }^{4}$, M. Besançon ${ }^{9}$, R. Beyer ${ }^{11}$, P. Biddulph ${ }^{22}$, J.C. Bizot ${ }^{27}$, V. Blobel ${ }^{13}$, K. Borras ${ }^{8}$, F. Botterweck ${ }^{4}$, V. Boudry ${ }^{28}$, A. Braemer ${ }^{14}$, F. Brasse ${ }^{11}$, W. Braunschweig ${ }^{1}$, V. Brisson ${ }^{27}$, D. Bruncko ${ }^{17}$, C. Brune ${ }^{15}$, R.Buchholz ${ }^{11}$, L. Büngener ${ }^{13}$, J. Bürger ${ }^{11}$, F.W. Büsser ${ }^{13}$, A. Buniatian ${ }^{11,39}$, S. Burke ${ }^{18}$, G. Buschhorn ${ }^{26}$, A.J. Campbell ${ }^{11}$,

T. Carli ${ }^{26}$, F. Charles ${ }^{11}$, D. Clarke ${ }^{5}$, A.B. Clegg ${ }^{18}$, B. Clerbaux ${ }^{4}$, M. Colombo ${ }^{8}$,

J.G. Contreras ${ }^{8}$, C. Cormack ${ }^{19}$, J.A. Coughlann ${ }^{5}$, A. Couraur ${ }^{27}$, Ch. Coutures ${ }^{9}$, G. Cozzika ${ }^{9}$,

L. Criegee ${ }^{11}$, D.G. Cussans ${ }^{5}$, J. Cvach ${ }^{30}$, S. Dagoret ${ }^{29}$, J.B. Dainton ${ }^{19}$, M. Danilov ${ }^{23}$,

W.D. Dau ${ }^{16}$, K. Daum ${ }^{34}$, M. David ${ }^{9}$, E. Deffur ${ }^{11}$, B. Delcourt ${ }^{27}$, L. Del Buono ${ }^{29}$,

A. De Roeck ${ }^{11}$, E.A. De Wolf ${ }^{4}$, P. Di Nezza ${ }^{32}$, C. Dollfus ${ }^{37}$, J.D. Dowell ${ }^{3}$, H.B. Dreis ${ }^{2}$,

V. Droutskoi ${ }^{23}$, J. Duboc ${ }^{29}$, D. Düllmann ${ }^{13}$, O. Dünger ${ }^{13}$, H. Duhm ${ }^{12}$, J. Ebert ${ }^{34}$,

T.R. Ebert ${ }^{19}$, G. Eckerlin ${ }^{11}$, V. Efremenko ${ }^{23}$, S. Egli ${ }^{37}$, H. Ehrlichmann ${ }^{35}$, S. Eichenberger ${ }^{37}$,

R. Eichler ${ }^{36}$, F. Eisele ${ }^{14}$, E. Eisenhandler ${ }^{20}$, R.J. Ellison ${ }^{22}$, E. Elsen ${ }^{11}$, M. Erdmann ${ }^{14}$,

W. Erdmann ${ }^{36}$, E. Evrard ${ }^{4}$, L. Favart ${ }^{4}$, A. Fedotov ${ }^{23}$, D. Feeken ${ }^{13}$, R. Felst ${ }^{11}$, J. Feltesse ${ }^{9}$,

J. Ferencei ${ }^{15}$, F. Ferrarotto ${ }^{32}$, K. Flamm ${ }^{11}$, M. Fleischer ${ }^{11}$, M. Flieser ${ }^{26}$, G. Flügge ${ }^{2}$,

A. Fomenko ${ }^{24}$, B. Fominykh ${ }^{23}$, M. Forbush ${ }^{7}$, J. Formánek ${ }^{31}$, J.M. Foster ${ }^{22}$, G. Franke ${ }^{11}$,

E. Fretwurst ${ }^{12}$, E. Gabathuler ${ }^{19}$, K. Gabathuler ${ }^{33}$, K. Gamerdinger ${ }^{26}$, J. Garvey ${ }^{3}$, J. Gayler ${ }^{11}$,

M. Gebaner ${ }^{8}$, A. Gellrich ${ }^{11}$, H. Genzel ${ }^{1}$, R. Gerhards ${ }^{11}$, U. Goerlach ${ }^{11}$, L. Goerlich ${ }^{6}$,

N. Gogitidze ${ }^{24}$, M. Goldberg ${ }^{29}$, D. Goldner ${ }^{8}$, B. Gonzalez-Pineiro ${ }^{29}$, I. Gorelov ${ }^{23}$,

P. Goritchev ${ }^{23}$, C. Grab ${ }^{36}$, H. Grässler ${ }^{2}$, R. Grässler ${ }^{2}$, T. Greenshaw ${ }^{19}$, G. Grindhammer ${ }^{26}$,

A. Gruber ${ }^{26}$, C. Gruber ${ }^{16}$, J. Haack ${ }^{35}$, D. Haidt ${ }^{11}$, L. Hajduk ${ }^{6}$, O. Hamon ${ }^{29}$, M. Hampe ${ }^{1}$,

E.M. Hanlon ${ }^{18}$, M. Hapke ${ }^{11}$, W.J. Haynes ${ }^{5}$, J. Heatherington ${ }^{20}$, G. Heinzelmann ${ }^{13}$,

R.C.W. Henderson ${ }^{18}$, H. Hensche ${ }^{35}$, R. Herma ${ }^{1}$, I. Herynek ${ }^{30}$, M.F. Hess ${ }^{26}$, W. Hildesheim ${ }^{11}$,

P. Hill ${ }^{5}$, K.H. Hiller ${ }^{35}$, C.D. Hilton ${ }^{22}$, J. Hladkýy $\dot{y}^{30}$, K.C. Hoeger ${ }^{22}$, M. Höppner ${ }^{8}$,

R. Horisberger ${ }^{33}$, Ph. Huet ${ }^{4}$, H. Hufnagel ${ }^{14}$, M. Ibbotson ${ }^{22}$, H. Itterbeck ${ }^{1}$, M.- A. Jabiol ${ }^{9}$,

A. Jacholkowska ${ }^{27}$, C. Jacobsson ${ }^{21}$, M. Jaffre ${ }^{27}$, J. Janoth ${ }^{15}$, T. Jansen ${ }^{11}$, L. Jönsson ${ }^{21}$,

K. Johannsen ${ }^{13}$, D.P. Johnson ${ }^{4}$, L. Johnson ${ }^{18}$, H. Jung ${ }^{29}$, P.I.P. Kalmus ${ }^{20}$, D. Kant ${ }^{20}$,

R. Kaschowitz ${ }^{2}$, P. Kasselmann ${ }^{12}$, U. Kathage ${ }^{16}$, H.H. Kaufmann ${ }^{35}$, S. Kazarian ${ }^{11}$,

I.R. Kenyon ${ }^{3}$, S. Kermiche ${ }^{25}$, C. Keuker ${ }^{1}$, C. Kiesling ${ }^{26}$, M. Klein ${ }^{35}$, C. Kleinwort ${ }^{13}$,

G. Knies ${ }^{11}$, W. Ko ${ }^{7}$, T. Köhler ${ }^{1}$, J. Köhne ${ }^{26}$, H. Kolanoski ${ }^{8}$, F. Kole ${ }^{7}$, S.D. Kolya ${ }^{22}$,

V. Korbel ${ }^{11}$, M. Korn ${ }^{8}$, P. Kostka ${ }^{35}$, S.K. Kotelnikov ${ }^{24}$, T. Krämerkämper ${ }^{8}$, M.W. Krasny ${ }^{6,29}$,

H. Krehbie ${ }^{11}$, D. Krücker ${ }^{2}$, U. Krüger ${ }^{11}$, U. Krüner-Marquis ${ }^{11}$, J.P. Kubenka ${ }^{26}$, H. Küster ${ }^{2}$,

M. Kuhlen ${ }^{26}$, T. Kurča ${ }^{17}$, J. Kurzhöfer ${ }^{8}$, B. Kuznik ${ }^{34}$, D. Lacour ${ }^{29}$, F. Lamarche ${ }^{28}$, R. Lander ${ }^{7}$, M.P.J. Landon ${ }^{20}$, W. Lange ${ }^{35}$, P. Lanius ${ }^{26}$, J.-F. Laporte ${ }^{9}$, A. Lebedev ${ }^{24}$, C. Leverenz ${ }^{11}$,

S. Levonian ${ }^{11,24}$, Ch. Ley ${ }^{2}$, A. Lindner ${ }^{8}$, G. Lindström ${ }^{12}$, F. Linse ${ }^{11}$, J. Lipinski ${ }^{13}$, B. List ${ }^{11}$,

P. $\operatorname{Loch}^{27}$, H. Lohmander ${ }^{21}$, G.C. Lopez ${ }^{20}$, V. Lubimove ${ }^{23}$, D. Lüke ${ }^{8,11}$, N. Magnussen ${ }^{34}$,

E. Malinovski ${ }^{24}$, S. Mani ${ }^{7}$, R. Maraček ${ }^{17}$, P. Marage ${ }^{4}$, J. Marks ${ }^{25}$, R. Marshalli22, J. Martens ${ }^{34}$,

R. Martin ${ }^{11}$, H.-U. Martyn ${ }^{1}$, J. Martyniak ${ }^{6}$, S. Masson ${ }^{2}$, T. Mavroidis ${ }^{20}$, S.J. Maxfield ${ }^{19}$,

S.J. McMahon ${ }^{19}$, A. Mehta ${ }^{22}$, K. Meier ${ }^{15}$, D. Mercer ${ }^{22}$, T. Merz ${ }^{11}$, C.A. Meyer ${ }^{37}$, H. Meyer ${ }^{34}$,

J. Meyer ${ }^{11}$, S. Mikocki ${ }^{6}$, D. Milstead ${ }^{19}$, F. Moreau ${ }^{28}$, J.V. Morris ${ }^{5}$, G. Müller ${ }^{11}$, K. Müller ${ }^{37}$,

P. Murín ${ }^{17}$, V. Nagovizin ${ }^{23}$, R. Nahnhauer ${ }^{35}$, B. Naroska ${ }^{13}$, Th. Naumann ${ }^{35}$, P.R. Newman ${ }^{3}$,

D. Newton ${ }^{18}$, D. Neyret ${ }^{29}$, H.K. Nguyen ${ }^{29}$, F. Niebergall ${ }^{13}$, C. Niebuhr ${ }^{11}$, R. Nisius ${ }^{1}$,

G. Nowak ${ }^{6}$, G.W. Noyes ${ }^{5}$, M. Nyberg-Werther ${ }^{21}$, M. Oakden ${ }^{19}$, H. Oberlack ${ }^{26}$, U. Obrock ${ }^{8}$,

J.E. Olsson ${ }^{11}$, E. Panaro ${ }^{12}$, A. Panitch ${ }^{4}$, C. Pascaud ${ }^{27}$, G.D. Patel ${ }^{19}$, E. Peppel ${ }^{35}$, E. Perez ${ }^{9}$,

J.P. Phillips ${ }^{22}$, Ch. Pichler ${ }^{12}$, D. Pitzl ${ }^{36}$, G. Pope $^{7}$, S. Prell ${ }^{11}$, R. Prosi ${ }^{11}$, G. Räde ${ }^{11}$,

F. Raupach ${ }^{1}$, P. Reimer ${ }^{30}$, S. Reinshagen ${ }^{11}$, P. Ribarics ${ }^{26}$, H.Rick ${ }^{8}$, V. Riech ${ }^{12}$,

J. Riedlberger ${ }^{36}$, S. Riess ${ }^{13}$, M. Rietz ${ }^{2}$, S.M. Robertson ${ }^{3}$, P. Robmann ${ }^{37}$, H.E. Roloff ${ }^{35}$,

R. Roosen ${ }^{4}$, K. Rosenbauer ${ }^{1}$ A. Rostovtsev ${ }^{23}$, F. Rouse ${ }^{7}$, C. Royon ${ }^{9}$, K. Rüter ${ }^{26}$, S. Rusakov ${ }^{24}$, 
K. Rybicki ${ }^{6}$, R. Rylko ${ }^{20}$, N. Sahlmann ${ }^{2}$, E. Sanchez ${ }^{26}$, D.P.C. Sankey ${ }^{5}$, M. Savitsky ${ }^{23}$, P. Schacht ${ }^{26}$, S. Schiek ${ }^{11}$, P. Schleper ${ }^{14}$, W. von Schlippe ${ }^{20}$, C. Schmidt ${ }^{11}$, D. Schmidt ${ }^{34}$, G. Schmidt ${ }^{13}$, A. Schöning ${ }^{11}$, V. Schröder ${ }^{11}$, E. Schuhmann ${ }^{26}$, B. Schwab ${ }^{14}$, A. Schwind ${ }^{35}$, U. Seehausen ${ }^{13}$, F. Sefkow ${ }^{11}$, M. Seide ${ }^{12}$, R. Sell ${ }^{11}$, A. Semenov ${ }^{23}$, V. Shekelyan ${ }^{23}$,

I. Sheviakov ${ }^{24}$, H. Shooshtari2 ${ }^{26}$, L.N. Shtarkov ${ }^{24}$, G. Siegmon ${ }^{16}$, U. Siewert ${ }^{16}$, Y. Sirois ${ }^{28}$, I.O. Skillicorn ${ }^{10}$, P. Smirnov ${ }^{24}$, J.R. Smith ${ }^{7}$, Y. Soloviev ${ }^{24}$, H. Spitzer ${ }^{13}$, R. Starosta ${ }^{1}$, M. Steenbock ${ }^{13}$, P. Steffen ${ }^{11}$, R. Steinberg ${ }^{2}$, B. Stella ${ }^{32}$, K. Stephens ${ }^{22}$, J. Stier ${ }^{11}$, J. Stiewe ${ }^{15}$, U. Stösslein ${ }^{35}$, J. Strachota ${ }^{30}$, U. Straumann ${ }^{37}$, W. Struczinski ${ }^{2}$, J.P. Sutton ${ }^{3}$, S. Tapprogge ${ }^{15}$, R.E. Taylor ${ }^{38,27}$, V. Tchernyshov ${ }^{23}$, C. Thiebaux ${ }^{28}$, G. Thompson ${ }^{20}$, P. Truöl ${ }^{37}$, J. Turnau ${ }^{6}$, J. Tutas ${ }^{14}$, P. Uelkes ${ }^{2}$, A. Usik ${ }^{24}$, S. Valkár ${ }^{31}$, A. Valkárová ${ }^{31}$, C. Vallée ${ }^{25}$, P. Van Esch $^{4}$, P. Van Mechelen ${ }^{4}$, A. Vartapetian ${ }^{11,39}$, Y. Vazdik ${ }^{24}$, M. Vecko ${ }^{30}$, P. Verrecchia ${ }^{9}$, G. Villet ${ }^{9}$,

K. Wacker ${ }^{8}$, A. Wagener ${ }^{2}$, M. Wagener ${ }^{33}$, I.W. Walker ${ }^{18}$, A. Walther ${ }^{8}$, G. Weber $^{13}$,

M. Weber ${ }^{11}$, D. Wegener ${ }^{8}$, A. Wegner ${ }^{11}$, H.P. Wellisch ${ }^{26}$, L.R. West ${ }^{3}$, S. Willard ${ }^{7}$, M. Winde ${ }^{35}$, G.-G. Winter ${ }^{11}$, A.E. Wright ${ }^{22}$, E. Wünsch ${ }^{11}$, N. Wulff ${ }^{11}$, T.P. Yiou ${ }^{29}$, J. Žáček ${ }^{31}$, D. Zarbock ${ }^{12}$, Z. Zhang ${ }^{27}$, A. Zhokin ${ }^{23}$, M. Zimmer ${ }^{11}$, W. Zimmermann ${ }^{11}$, F. Zomer ${ }^{27}$ and K. Zuber ${ }^{15}$

${ }^{1}$ I. Physikalisches Institut der RWTH, Aachen, Germany ${ }^{a}$

${ }^{2}$ III. Physikalisches Institut der RWTH, Aachen, Germany ${ }^{a}$

${ }^{3}$ School of Physics and Space Research, University of Birmingham, Birmingham, $U K^{b}$

${ }^{4}$ Inter-University Institute for High Energies ULB-VUB, Brussels; Universitaire Instellingen Antwerpen, Wilrijk, Belgium ${ }^{e}$

${ }^{5}$ Rutherford Appleton Laboratory, Chilton, Didcot, $U K^{b}$

${ }^{6}$ Institute for Nuclear Physics, Cracow, Poland ${ }^{d}$

${ }^{7}$ Physics Department and IIRPA, University of California, Davis, California, USA ${ }^{e}$

${ }^{8}$ Institut für Physik, Universität Dortmund, Dortmund, Germany ${ }^{a}$

${ }^{9}$ CEA, DSM/DAPNIA, CE-Saclay, Gif-sur-Yvette, France

${ }^{10}$ Department of Physics and Astronomy, University of Glasgow, Glasgow, UK ${ }^{b}$

11 DESY, Hamburg, Germany ${ }^{a}$

${ }^{12}$ I. Institut für Experimentalphysik, Universität Hamburg, Hamburg, Germany ${ }^{a}$

${ }^{13}$ II. Institut für Experimentalphysik, Universität Hamburg, Hamburg, Germany ${ }^{a}$

${ }^{14}$ Physikalisches Institut, Universität Heidelberg, Heidelberg, Germany ${ }^{a}$

${ }^{15}$ Institut für Hochenergiephysik, Universität Heidelberg, Heidelberg, Germany ${ }^{a}$

${ }^{16}$ Institut für Reine und Angewandte Kernphysik, Universität Kiel, Kiel, Germany ${ }^{a}$

17 Institute of Experimental Physics, Slovak Academy of Sciences, Košice, Slovak Republic

18 School of Physics and Materials, University of Lancaster, Lancaster, UK ${ }^{b}$

19 Department of Physics, University of Liverpool, Liverpool, UK ${ }^{b}$

${ }^{20}$ Queen Mary and Westfield College, London, UK $K^{b}$

${ }^{21}$ Physics Department, University of Lund, Lund, Sweden ${ }^{f}$

${ }^{22}$ Physics Department, University of Manchester, Manchester, UK ${ }^{b}$

${ }^{23}$ Institute for Theoretical and Experimental Physics, Moscow, Russia

${ }^{24}$ Lebedev Physical Institute, Moscow, Russia

${ }^{25}$ CPPM, Université d'Aix-Marseille II, IN2P3-CNRS, Marseille, France

${ }^{26}$ Max-Planck-Institut für Physik, München, Germany ${ }^{a}$

${ }^{27}$ LAL, Université de Paris-Sud, IN2P3-CNRS, Orsay, France

${ }^{28}$ LPNHE, Ecole Polytechnique, IN2P3-CNRS, Palaiseau, France

${ }^{29}$ LPNHE, Universités Paris VI and VII, IN2P3-CNRS, Paris, France

${ }^{30}$ Institute of Physics, Czech Academy of Sciences, Praha, Czech Republic ${ }^{g}$

${ }^{31}$ Nuclear Center, Charles University, Praha, Czech Republic ${ }^{g}$

${ }^{32}$ INFN Roma and Dipartimento di Fisica, Universita "La Sapienza", Roma, Italy

${ }^{33}$ Paul Scherrer Institut, Villigen, Switzerland

${ }^{34}$ Fachbereich Physik, Bergische Universität Gesamthochschule Wuppertal, Wuppertal, Germany ${ }^{a}$ 
${ }^{35}$ DESY, Institut für Hochenergiephysik, Zeuthen, Germany ${ }^{a}$

${ }^{36}$ Institut für Teilchenphysik, ETH, Zürich, Switzerland ${ }^{\text {h }}$

37 Physik-Institut der Universität Zürich, Zürich, Switzerland ${ }^{h}$

38 Stanford Linear Accelerator Center, Stanford California, USA

39 Visitor from Yerevan Phys.Inst., Armenia

a Supported by the Bundesministerium für Forschung und Technologie, FRG under contract numbers 6AC17P, 6AC47P, 6DO57I, 6HH17P, 6HH27I, 6HD17I, 6HD27I, 6KI17P, 6MP17I, and $6 \mathrm{WT} 87 \mathrm{P}$

${ }^{b}$ Supported by the UK Particle Physics and Astronomy Research Council, and formerly by the

UK Science and Engineering Research Council

c Supported by FNRS-NFWO, IISN-IIKW

¿ Supported by the Polish State Committee for Scientific Research, grant No. 204209101

${ }^{\circ}$ Supported in part by USDOE grant DE F603 91ER 40674

${ }^{f}$ Supported by the Swedish Natural Science Research Council

g Supported by GA $\breve{C} R$, grant no. 202/93/2423 and by GA AV $\check{C} R$, grant no. 19095

${ }^{h}$ Supported by the Swiss National Science Foundation 


\section{Introduction}

In perturbative Quantum Chromodynamics (QCD), the theory of strong interactions at short distances, cross sections are expressed as power series in the "running coupling constant" $\alpha_{s}$. The value of this fundamental parameter is not given by the theory itself but has to be determined from experiment. Much experimental effort has gone into the investigation of the dependence of $\alpha_{s}\left(\mu^{2}\right)$ on the renormalization scale $\mu^{2}$ as predicted by the renormalization group equation (RGE [1]) and the determination of its value at a particular scale, say $\mu^{2}=M_{Z}^{2}$, the mass squared of the neutral heavy vector boson.

The study of the relative number of events with jets initiated by the emission of an extra gluon from the outgoing $q \bar{q}$ pair is a method used commonly to determine $\alpha_{s}$ in $e^{+} e^{-}$annihilation, because this observable is known to have small corrections due to soft hadronic physics [2]. It is not a priori clear that a similar procedure can be applied in deep inelastic ep scattering (DIS). The main reason is that, in contrast to $e^{+} e^{-}$annihilation, the strongly interacting partons are already present in the initial state. Besides the problem of dealing with the influence of the hadronization mechanism, one expects significant contributions from multiple gluon emission and quark pair creation particularly in the initial state. These parton shower processes are usually only modeled in QCD-inspired Monte Carlo calculations and are not understood at a more fundamental level. Also the parton density functions (PDF) inside the proton are not precisely known.

In this paper a first attempt is made to extract $\alpha_{s}$ from jet multiplicities in DIS. Jet production rates at the partonic level are obtained from jet rates at the detector level by the application of a correction factor and are compared to theoretical predictions in next to leading order (NLO). The paper is organized as follows. Following a discussion of the theoretical basis, the H1-experiment and the data selection are described. Then the correction factors relating the observed jets in the detector to the underlying parton jets and the restrictions in phase space which minimize the influence of parton showers are discussed. Finally the extraction of $\alpha_{s}$ from the data is described.

Many experimental questions relevant to this study, e.g. the existence and properties of jets in DIS, the applicability of the JADE jet clustering algorithm [3], the comparison to QCD Monte Carlo models etc., have already been discussed in a previous paper [4]. Other jet studies with comparisons to leading order ( $\mathrm{LO}$ ) QCD calculations can be found in $[5,6]$.

\section{Theoretical Basis}

The simplest process leading to jets in the final state in DIS is the hard scattering reaction $e+q \rightarrow e^{\prime}+q^{\prime}$. In order to explicitly account for the proton remnant this process is called a $(1+1)$-jet reaction. A $(2+1)$-jet reaction arises in QCD at order $\mathcal{O}\left(\alpha_{s}\right)$ via the emission of a hard gluon from the initial or final state quark (QCD Compton process) or via $q \bar{q}$ production in photon gluon fusion processes.

Identifying the scale $\mu^{2}$ with the absolute value of the square of the four momentum transfer $Q^{2}$ from the incident electron, the ratio of jet cross sections

$$
R_{2+1}\left(Q^{2}\right)=\frac{\sigma_{2+1}\left(Q^{2}\right)}{\sigma_{\text {tot }}\left(Q^{2}\right)}
$$

is determined by the value of $\alpha_{s}\left(Q^{2}\right)$. The symbol $\sigma_{\text {tot }}$ here abbreviates the sum $\sigma_{1+1}+\sigma_{2+1}$.

In leading $\log$ QCD calculations, the cut-off parameter $\Lambda$ in the expression for $\alpha_{S}$ is not well defined. Changing $\Lambda$ to $\Lambda^{\prime}$ is equivalent to replacing $\log \left(Q^{2} / \Lambda^{2}\right)$ by $\log \left(Q^{2} / \Lambda^{\prime 2}\right)+2 \log \left(\Lambda^{\prime} / \Lambda\right)$. In 
leading $\log$ all constant terms are dropped and therefore all $\Lambda$ values are equivalent. In contrast, in two loop calculations the constant terms are fixed and $\Lambda$ becomes a meaningful quantity. For quantitative comparisons of the experimental rates with QCD which allow a determination of $\alpha_{s}$ it is therefore mandatory to have also $R_{2+1}$ computed to $\mathcal{O}\left(\alpha_{s}^{2}\right)$ i.e. in NLO. In this paper we use the results of D. Graudenz [7, 8], who investigated jet production in electron proton scattering mediated via the exchange of virtual photons. The $(1+1)$ and the $(2+1)$-cross sections are computed for all photon helicity amplitudes up to NLO. Numerical predictions of the cross sections for different experimental configurations based on these calculations can be made using the Monte Carlo program PROJET [9]. Other NLO calculations evaluating the sum over the polarization states of the virtual photon have also been published [10].

In NLO the respective cross sections are given by

$$
\sigma_{1+1}\left(Q^{2}, y_{c}\right)=A_{1+1,0}\left(Q^{2}\right)+\alpha_{s}\left(Q^{2}\right) A_{1+1,1}\left(Q^{2}, y_{c}\right)
$$

and

$$
\sigma_{2+1}\left(Q^{2}, y_{c}\right)=\alpha_{s}\left(Q^{2}\right) A_{2+1,1}\left(Q^{2}, y_{c}\right)+\alpha_{s}^{2}\left(Q^{2}\right) A_{2+1,2}\left(Q^{2}, y_{c}\right)
$$

The terms $A_{i, j}$ contain the hard scattering matrix elements (without the strong coupling constant) and the parton densities of the incoming proton. The first index stands for the jet multiplicity as defined above. The second index indicates the order $\alpha_{s}^{j}$ to which the process is calculated. $y_{c}$ is a jet resolution parameter (defined below), which is necessary both for the assignment of final states with soft and nearly collinear partons to a given cross section class and for the regularization of infinities in the theoretical expressions. Using eqs. (2) and (3) the ratio in eq. (1) can also be expressed as a power series in $\alpha_{s}$ which is correct to $\mathcal{O}\left(\alpha_{s}^{2}\right)$, i.e. only $(1+1)$ and $(2+1)$-jet events have to be included in the calculation of $\sigma_{t o t}$.

The parton densities contained in $A_{i, j}$ are calculated at the factorization scale $Q^{2}$. They are usually obtained via evolution from a low lying scale $Q_{0}^{2}$ using a value $\alpha_{s}\left(M_{Z}^{2}\right)$ as input parameter, which may differ from that obtained in this analysis. For strict consistency one would have to re-evolve the distributions using the value of $\alpha_{s}\left(M_{Z}^{2}\right)$ obtained here [11]. Given the presently obtainable accuracy of measurement (see section 6.2) this is however not necessary.

Besides the arguments given explicitly above, the functions $A_{i, j}$ also depend on other experimental parameters such as acceptance cuts for the scattered electron and the jets. The numerator of eq. (1) is computed from all configurations with two jets inside the acceptance region and events with at least one jet in the acceptance region contribute to the denominator.

In PROJET the outgoing partons are grouped into jets using a modified JADE cluster algorithm, where the proton remnant is represented by a pseudoparticle which carries only longitudinal momentum. The algorithm thus automatically checks whether an outgoing parton can be resolved from the remnant. The jet resolution parameter is given by

$$
y_{c}=\frac{m_{i j}^{2}}{W^{2}}
$$

where $W$ is the invariant mass of the hadronic system and $m_{i j}$ the invariant mass of any two objects including the pseudoparticle. The choice of the scale $W$ ensures a treatment of the jet classification in DIS analogous to that used in $e^{+} e^{-}$annihilation and is also preferred for other theoretical reasons [12].

\section{$3 \quad$ H1 Apparatus}

A detailed discussion of the $\mathrm{H} 1$ apparatus may be found elsewhere [13]. Here emphasis is put on describing the main features of the components of the detector relevant to this analysis, 
which makes use mainly of the calorimeters and, to a lesser extent, of the central and backward tracking systems. Polar angles $\theta$ are defined with respect to the proton beam direction.

The inner part of the detector consists of the central tracking chamber (CT) supplemented by a forward tracking detector (FT) and a backward proportional chamber (BPC), covering the polar angle ranges $25^{\circ}<\theta<155^{\circ}, 7^{\circ}<\theta<25^{\circ}$ and $155^{\circ}<\theta<175^{\circ}$, respectively. The central and forward tracking devices are used to determine the vertex position, which is for $95 \%$ of the events within $30 \mathrm{~cm}$ of the nominal interaction point. The $\mathrm{BPC}$, together with the vertex, is used to measure the electron scattering angle in the backward region. The angular resolution achieved is better than 2 mrad.

The scattered electron and the hadronic energy flow are measured in a liquid argon (LAr) calorimeter and in a backward electromagnetic lead-scintillator calorimeter (BEMC). Hadronic showers which are not contained in the LAr are measured in a surrounding instrumented iron system housed in the return yoke of the superconducting solenoid. The solenoid is outside the LAr calorimeter, and it provides a uniform magnetic field of $1.15 \mathrm{~T}$ parallel to the proton beam axis at the interaction point.

The LAr calorimeter [14] extends over the polar angle range $4^{\circ}<\theta<153^{\circ}$ with full azimuthal coverage. The calorimeter consists of an electromagnetic section with lead absorbers, corresponding to a depth of between 20 and 30 radiation lengths, and a hadronic section with steel absorbers. The total depth of the LAr calorimeter varies between 4.5 and 8 hadronic interaction lengths. The calorimeter is highly segmented in both sections with a total of around 45000 geometric cells. The electronic noise per channel is typically in the range 10 to $30 \mathrm{MeV}$ ( $1 \sigma$ equivalent energy). Test beam measurements of LAr calorimeter modules have demonstrated energy resolutions of $\sigma(E) / E \approx 0.12 / \sqrt{E / \mathrm{GeV}} \oplus 0.01$ for electrons [15] and $\sigma(E) / E \approx 0.5 / \sqrt{E / \mathrm{GeV}} \oplus 0.02$ for charged pions $[13,16]$. The hadronic energy scale and resolution have been verified from the balance of transverse momentum between hadronic jets and the scattered electron in DIS events and are known to a precision of $5 \%$ and $10 \%$ respectively. The uncertainty of the absolute energy scale for electrons is at the level of $3 \%$.

The BEMC, with a thickness of 22.5 radiation lengths, covers the backward region of the detector, $151^{\circ}<\theta<177^{\circ}$. It is mainly used to trigger on and to measure electrons from DIS processes at low $Q^{2}$. The acceptance region corresponds to $Q^{2}$ values in the approximate range $5 \leq Q^{2} \leq 100 \mathrm{GeV}^{2}$. A resolution of $\sigma(E) / E \approx 0.10 / \sqrt{E / \mathrm{GeV}} \oplus 0.03$ has been achieved. By adjusting the measured electron energy spectrum to the kinematic peak the BEMC energy scale is known to an accuracy of $1.7 \%$.

A scintillator hodoscope situated behind the BEMC is used to veto proton-induced background events based on their early time of arrival compared to that of the nominal electron proton collision.

\section{Trigger and Data Selection}

The analysis is based on a sample of DIS events taken in 1993 with an integrated luminosity of $\approx 0.3 \mathrm{pb}^{-1}$. Because of the nature of the $\mathrm{H} 1$ detector the data are divided into two sub-samples with different sources of systematic errors. The sub-samples consist of events with the scattered electron detected in the BEMC and in the LAr calorimeter respectively. In both cases $Q^{2}$ and the usual DIS scaling variable $y$ are calculated from the energy and angle of the scattered electron.

The hardware trigger of the first sub-sample requires an energy cluster with more than $4 \mathrm{GeV}$ in the BEMC and no time of flight veto from the scintillator hodoscope. After reconstruction the data are subject to the following selection criteria: 
1. An electromagnetic cluster must be present in the BEMC, which matches a space point in the $\mathrm{BPC}$, in the range $160^{\circ}<\theta_{e}<172.5^{\circ}$.

2. The center of gravity of the electron cluster must have transverse distances to the beam $>14 \mathrm{~cm}$ in either of two orthogonal directions to assure that the electron is well contained inside the BEMC.

3. The four-momentum transfer $Q^{2}$ measured using the electron must be between 10 and 100 $\mathrm{GeV}^{2}$.

4. The energy $E_{e}^{\prime}$ of the scattered electron must be greater than $14 \mathrm{GeV}$, corresponding to $y \lesssim 0.5$, thus eliminating background from photoproduction. By also suppressing radiative DIS events this cut strongly reduces radiative corrections to the cross section.

The second sub-sample consists of events in which the scattered electron is fully contained in the LAr system. These events were triggered by the LAr electron trigger. Energy clusters are reconstructed from the calorimeter cells. The electromagnetic cluster with the highest transverse energy is accepted as the scattered electron after the following additional cuts:

1. $Q^{2}>100 \mathrm{GeV}^{2}$.

2. $y<0.7$ to suppress photoproduction and radiative DIS events.

3. $10^{\circ}<\theta_{e}<148^{\circ}$ to ensure the electron is contained in the LAr calorimeter and to avoid the transition region between LAr and BEMC.

4. No muon track is allowed within a cone of $5^{\circ}$ half opening angle around the direction of the electron in the instrumented iron system in order to remove cosmic ray events.

5. The energy deposited in the electromagnetic calorimeter in a cylinder extending from radius $15 \mathrm{~cm}$ to $30 \mathrm{~cm}$ around the electron direction must be below $1.2 \mathrm{GeV}$ to ensure that the electron is isolated. Similarly the energy in the hadronic calorimeter within 30 $\mathrm{cm}$ around the electron direction must be less than $0.5 \mathrm{GeV}$.

6. To avoid migrations from low $Q^{2}$ events into the LAr sample, the maximum BEMC cluster energy allowed is $10 \mathrm{GeV}$.

7. The quantity $\delta=\sum_{\text {clusters }}\left(E-P_{z}\right)$ is limited to $30 \mathrm{GeV}<\delta<70 \mathrm{GeV}$. The lower cut excludes events from photoproduction with the scattered electron inside the beam pipe. As does the cut in $y$ discussed above, this cut also reduces radiative corrections to the cross section.

The following additional requirements were imposed on both sub-samples:

1. An event vertex within $\pm 30 \mathrm{~cm}$ in $z$ of the nominal interaction point.

2. $W^{2}>5000 \mathrm{GeV}^{2}$. This cut ensures an invariant mass squared of the two hard jets $>100 \mathrm{GeV}^{2}$ for $(2+1)$-jet events at $y_{c}>0.02$. $W^{2}$ is calculated using the double angle method [17]. This method relies on the angle of the scattered electron and the angle of the total hadronic system and not on the jet multiplicity.

The final sample contains 12485 events with $Q^{2}<100 \mathrm{GeV}^{2}$ and 737 events with $Q^{2}>100$ $\mathrm{GeV}^{2}$. A fraction of $6.6 \%$ in the low $Q^{2}$ sample and of $4.2 \%$ in the high $Q^{2}$ sample is compatible with being so called rapidity gap events. They were defined by the requirement that the distance in pseudorapidity $\eta$ between the most forward calorimeter cluster with energy $>400 \mathrm{MeV}$ and the most forward LAr calorimeter cell exceeds 1.8. These events have been shown to depend on $Q^{2}$ and the Bjorken variable $x$ rather similarly to the other DIS events [18]. They are therefore not treated separately. 


\section{Jet Analysis}

Jets are reconstructed from energy clusters in the calorimeters using the JADE algorithm with the remnant pseudoparticle represented by the missing longitudinal momentum in the detector. This choice of algorithm is imposed because the theoretical calculations [7,8] are performed in this scheme. Here $W$ needed in eq. (4) is calculated from the invariant mass of all objects used including the pseudoparticle. The rates $R_{N+1}$ are calculated from the number of events with $N$ jets inside the acceptance region divided by the number of events with at least one jet inside this region.

In our previous publication [4] it was shown that the jet rates $R_{N+1}$ as a function of $y_{c}$ are very well described by the LEPTO 6.1 Monte Carlo program [19] using the MEPS model. This model is based on LO matrix elements and an approximate treatment of higher order effects with leading log parton showers in the initial and final state. The hadronization follows the Lund string model as implemented in JETSET [20]. Using this model it was demonstrated that the jet rates at the detector level and the parton level agree within $15 \%$ for $y_{c}>0.02$ and that there is a moderate migration between jet classes due to the finite resolution in $m_{i j}^{2}$. For example $\approx 60 \%$ of the $(2+1)$-jet events at the parton level remain in this class at the detector level, a value similar to that observed in $e^{+} e^{-}$annihilation experiments [21].

The present analysis with much higher statistics confirms all these findings. The good description of the data obtained using the LEPTO generator is however strongly dependent on the inclusion of parton showers in the MEPS model. The $(2+1)$-jet rate at $y_{c}=0.02$ is increased by more than $50 \%$ compared to a LO matrix element calculation if parton showers are included. This large effect is mainly due to initial state parton showering and cannot be accounted for by going from LO to NLO matrix elements.

Using the MEPS model it was found that a cut on the jet polar angle in the laboratory system $\left(\vartheta_{j e t}>10^{\circ}\right)$ greatly reduces the influence of parton showers, particularly those from the initial state. With this cut the $(2+1)$-jet rate with and without inclusion of parton showers differs by less than $10 \%$. Because the BEMC is not designed for the measurement of hadronic jets, the jet angle in the backward direction is restricted to $\vartheta_{\text {jet }}<145^{\circ}$. The MEPS model with these additional cuts gives a very good description of the $R_{N+1}$ distributions in the LAr sample for all values of $y_{c}$. The BEMC sample is fairly well described for $y_{c}>0.015$ (fig. 1).

In order to determine $\alpha_{s}\left(Q^{2}\right)$ the low $Q^{2}$ sample is divided into three bins with about equal populations of $(2+1)$-jet events, and the high $Q^{2}$ sample into two bins. $R_{2+1}$ at the parton level is obtained by multiplying $R_{2+1}$ at the detector level by a correction factor. Without acceptance cuts in $\vartheta_{\text {jet }}$ these factors were found to be close to unity. The inclusive $\vartheta_{\text {jet }}$ distribution at the parton level differs, however, from that at the detector level, especially at low values of $Q^{2}$. Therefore, the $\vartheta_{\text {jet }}$ cut needed for the suppression of parton showers leads to increased correction factors, which were calculated using high statistics Monte Carlo sets generated with the MRSD ${ }^{-}$ parton densities [22]. They range between 1.08 and 1.52 and are given in table 1 (column 1). The quoted errors are due to the Monte Carlo statistics.

The correction factors were also calculated using a Monte Carlo model which leads to a different partonic configuration in the final state. Events were generated using LEPTO 6.1 to simulate the electroweak interaction and photon gluon fusion, followed by ARIADNE 4.03 [23] which includes QCD Compton and further gluon emission in the framework of the colour dipole model. Such event simulations are henceforth referred to as CDM. For more details see ref. [4]. The MRSH parton density parametrization [24], which has been derived from a fit to the HERA structure function measurements [25], was used. It is similar to the older $\mathrm{MRSD}^{-}$parametrization. The CDM model describes the jet rates as a function of $y_{c}$ at high $Q^{2}$ with an accuracy similar to the MEPS model. The measured $y_{c}$ dependence at low $Q^{2}$ and the $Q^{2}$ dependence 

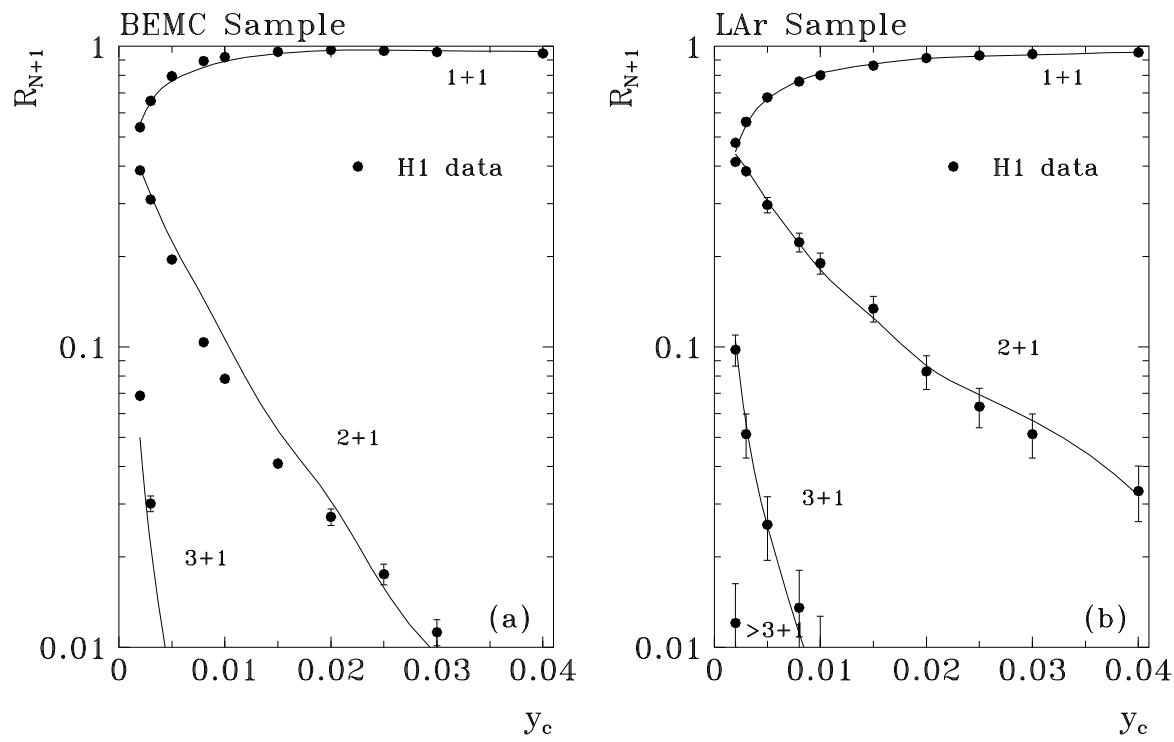

Figure 1: The $R_{N+1}$ distribution compared to the MEPS model for jets with $10^{\circ}<\vartheta_{\text {jet }}<145^{\circ}$ for a) $Q^{2}<100 \mathrm{GeV}^{2}$ and b) $Q^{2}>100 \mathrm{GeV}^{2}$. The points are the observed jet rates with statistical errors only. The full line represents the Monte Carlo prediction at the detector level. The parton density parametrization used is $\mathrm{MRSD}^{-}$.

of $R_{2+1}$ is however not as well described by this model. The correction factors are considerably bigger than in the MEPS model, and the differences decrease with increasing $Q^{2}$ (table 1 , column 2).

\begin{tabular}{c|c|c}
\hline \hline$Q^{2}\left[\mathrm{GeV}^{2}\right]$ & MEPS & CDM \\
\hline $10-18$ & $1.08 \pm 0.10$ & $2.13 \pm 0.12$ \\
\hline $18-30$ & $1.52 \pm 0.19$ & $2.10 \pm 0.15$ \\
\hline $30-100$ & $1.46 \pm 0.17$ & $2.19 \pm 0.17$ \\
\hline $100-400$ & $1.38 \pm 0.13$ & $1.68 \pm 0.06$ \\
\hline $400-4000$ & $1.10 \pm 0.11$ & $1.28 \pm 0.06$ \\
\hline \hline
\end{tabular}

Table 1: Correction factors needed to calculate $R_{2+1}$ at parton level from $R_{2+1}$ at detector level for two different Monte Carlo models.

\section{$6 \quad$ Results}

\subsection{Comparison of $R_{2+1}$ to the NLO Calculation}

In fig. 2a the corrected rates, as defined in eq. (1), are plotted as a function of $Q^{2}$ for $y_{c}=0.02$. The correction factors are taken from the MEPS model as this provides the best description of the data. The errors shown arise from the statistical error of the data and that of the correction 
factor described above. The data are compared to the PROJET $\mathcal{O}\left(\alpha_{s}^{2}\right)$ prediction using the MRSH parton densities. The QCD cutoff parameter $\Lambda$ is varied between 200 and $500 \mathrm{MeV}$, the range allowed by previous measurements [2]. The $\Lambda$ symbol is taken as an abbreviation for $\Lambda \frac{(4)}{M S}[26]$. The data are well described by a fundamental QCD calculation over a wide range of space like momentum transfer squared.

Following the prescription in section 2, the strong coupling constant has been simultaneously determined for 5 different values of $Q^{2}$. The result (fig. $2 \mathrm{~b}$ ) shows clearly the decrease of $\alpha_{s}$ with the renormalization scale as predicted by the RGE. The falling dashed line represents the fit obtained from a $\chi^{2}$ minimization of the data to the QCD prediction $\left(\chi^{2} /\right.$ dof $\left.=1.39\right)$. In addition a fit to the Ansatz of constant $\alpha_{s}$ is also included in the figure. This assumption is clearly in conflict with the data $\left(\chi^{2} /\right.$ dof $\left.=5.1\right)$.
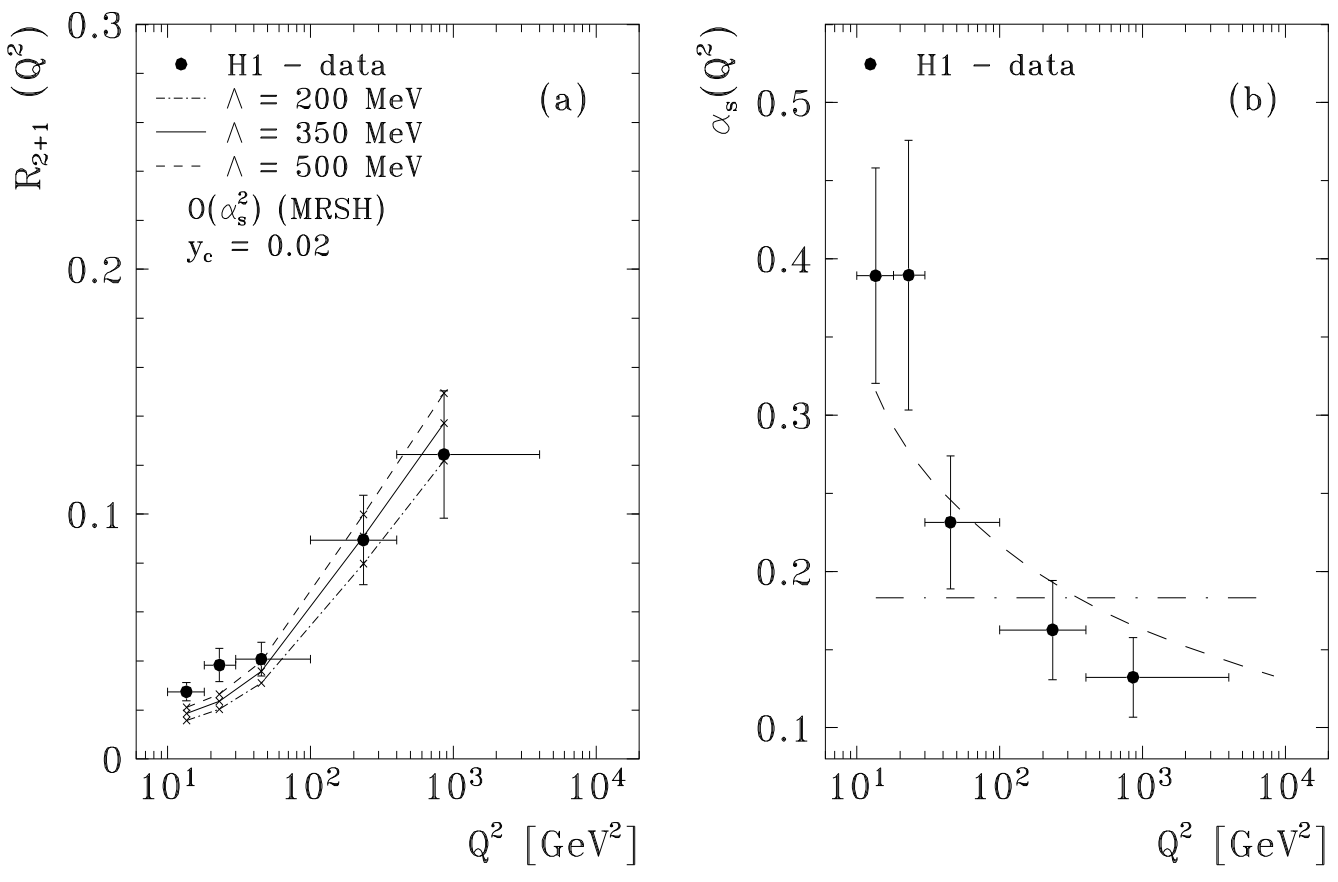

Figure 2: Comparison of corrected jet rates to QCD in next to leading order. The vertical error bars correspond to the statistical error of the data and the correction factors. a) The ratio $R_{2+1}$ at $y_{c}=0.02$ as a function of $Q^{2}$ together with the PROJET prediction for various $\Lambda$ values and the MRSH parton density. b) The measured value of $\alpha_{s}$ as a function of $Q^{2}$. The fit to the RGE prediction (falling dashed curve) is shown. For comparison the fit to the Ansatz of constant $\alpha_{s}$ is also included.

The main result of figs. $2 \mathrm{a}$ and $2 \mathrm{~b}$, namely the good description of the jet rates by aCD calculation in NLO and the evidence for the running of $\alpha_{s}$, is within the given model for the calculation of correction factors rather insensitive to the details of the final analysis, for example the choice of the parton density parametrizations and the value of the $\vartheta_{j e t}$ cut [27]. However the fit result for $\alpha_{S}\left(M_{Z}^{2}\right)$ depends critically on the inclusion of the low $Q^{2}$ data, for which the dependence on both the PDF and the cut in $\vartheta_{\text {jet }}$ is strongest. The systematic uncertainty of the 
two lowest $Q^{2}$ entries has been estimated by selecting different PDF and varying the $\vartheta_{\text {jet }}$ cut $^{1}$. It was found to be less than $\pm 20 \%$. In addition, at low $Q^{2}$ the correction factors are very model dependent (table 1). Therefore only the two entries from the high $Q^{2}$ sample are considered in the following.

\subsection{Determination of $\alpha_{s}\left(M_{Z}^{2}\right)$}

A QCD fit to the two high $Q^{2}$ measurements of $\alpha_{s}$ in fig. $2 \mathrm{~b}$ yields

$$
\alpha_{s}\left(M_{Z}^{2}\right)=0.115 \pm 0.012
$$

with $\chi^{2}=0.09$ per degree of freedom. The quoted error includes only the statistical error from data and Monte Carlo.

Although the color dipole model does not give a good description of the measured jet rates in the low $Q^{2}$ region it is used to estimate the model dependence of the fit result for the high $Q^{2}$ sample. Using the correction factors from this model yields $\alpha_{S}\left(M_{Z}^{2}\right)=0.130 \pm 0.012$. From the average of both fits $\alpha_{S}\left(M_{Z}^{2}\right)=0.123 \pm 0.012 \pm 0.008$ is derived. The first error is statistical, and the second due to the model dependence of the correction factors.

In order to estimate further systematic errors of the analysis several checks have been made [27] using the MEPS model for calculating the correction factors. They are discussed in the following and the results are included in table 2.

For $(2+1)$-jet events the fraction $z$ of the initial state proton momentum taken by the incoming parton is given by

$$
z=y_{c}+x\left(1-y_{c}\right),
$$

where $x$ is the usual Bjorken scaling variable. Therefore, with $y_{c}=0.02$, the numerator in eq.(1) only contains events with $z>0.02$, whereas the denominator is dominated by events with $z$ values reaching down to $1.5 \times 10^{-3}$ where the quark densities are not so well constrained. To account for this uncertainty, the QCD fit was repeated using CTEQ(2pM) [28] which also includes the HERA structure function measurements. The fit yields $\alpha_{s}\left(M_{Z}^{2}\right)=0.117 \pm 0.012$.

Around $50 \%$ of the $(2+1)$-jet cross section at high $Q^{2}$ is gluon initiated. Repeating the analysis with the GRV parametrization [29], which contains distinctly more gluons at small $z$ values, $\alpha_{s}\left(M_{Z}^{2}\right)=0.114 \pm 0.012$ is obtained. In addition an overall uncertainty of $\pm 10 \%$ in the gluon density of MRSH was assumed. This error band is in accordance with a recent investigation by the NMC collaboration [30] at $Q^{2}<20 \mathrm{GeV}^{2}$ in which the systematic errors of the gluon density and the sensitivity to the input value of $\alpha_{s}$ were determined. The resulting error in $\alpha_{s}\left(M_{Z}^{2}\right)$ due to the combined uncertainty in the quark and gluon densities was thus calculated to be \pm 0.005 .

For an investigation of the systematic uncertainty resulting from the restricted range of jet angles, the lower $\vartheta_{j e t}$ cut was varied between $8^{\circ}$ and $12^{\circ}$ and the upper cut between $140^{\circ}$ and $150^{\circ}$. From the observed dependence of $\alpha_{s}\left(M_{Z}^{2}\right)$ on the value of the $\vartheta_{j e t}$ cut a further systematic error of \pm 0.003 was calculated.

Due to the finite resolution in $m_{i j}^{2}$, the result also depends on the chosen value of the jet resolution parameter $y_{c}$. Repeating the analysis for $y_{c}=0.03$, the systematic error due to the particular choice of $y_{c}=0.02$ was estimated to be \pm 0.005 .

Since $m_{i j}^{2} / W^{2}$ depends linearly on the hadronic energy, an error in the calorimetric energy scale influences the jet classification at fixed $y_{c}$ and therefore our determination of $\alpha_{s}$. This scale is presently known to an accuracy of $5 \%$ leading to $\Delta \alpha_{s}\left(M_{Z}^{2}\right)= \pm 0.006$.

\footnotetext{
${ }^{1}$ A detailed discussion follows in the next section.
} 


\begin{tabular}{c|c}
\hline \hline Error source & $\Delta \alpha_{s}\left(M_{Z}^{2}\right)$ \\
\hline QCD models & \pm 0.008 \\
\hline Parton density functions & \pm 0.005 \\
\hline$\vartheta_{\text {jet }}$ cut & \pm 0.003 \\
\hline Value of $y_{c}$ & \pm 0.005 \\
\hline Renormalization and factorization scale & \pm 0.006 \\
\hline
\end{tabular}

Table 2: Systematic errors on the determination of $\alpha_{s}\left(M_{Z}^{2}\right)$

The default renormalization scale assumed by PROJET is $Q^{2}$. By varying the scale in multiples of $Q^{2}$ as suggested in [8] A the uncertainty in $\alpha_{s}\left(M_{Z}^{2}\right)$ due to this scale was found to be \pm 0.002 for scales between $Q^{2} / 4$ and $4 Q^{2}$. By changing the factorization scale $Q^{2}$ in the same range the uncertainty due to the factorization scale in the PDF was proven to be of the same order. The investigation of other proposed scales [31] is left to a future analysis.

Adding the statistical error and the systematic errors of table 2 in quadrature yields

$$
\alpha_{s}\left(M_{z}^{2}\right)=0.123 \pm 0.018
$$

This has to be compared with $\alpha_{s}=0.119 \pm 0.010$ obtained from the LEP experiments using the same observable in NLO [2], and with $\alpha_{s}\left(M_{Z}^{2}\right)=0.117 \pm 0.005$ from the world average [32].

\section{Conclusions}

It has been shown that the jet rates in deep inelastic electron proton scattering are well described by a second order QCD calculation. Dividing the data into five $Q^{2}$ bins, the coupling constant $\alpha_{s}$ was measured, though in a model dependent way, at these different scales using one observable in a single experiment. For $Q^{2}$ values $>100 \mathrm{GeV}^{2}$, the dependence on correction factors obtained in different QCD models is much reduced, permitting a determination of $\alpha_{S}\left(M_{Z}^{2}\right)=0.123 \pm 0.018$. The agreement between the $\alpha_{s}$ values determined from the same observable in deep inelastic $e p$ scattering and $e^{+} e^{-}$annihilation again demonstrates the coherence and consistency of the underlying QCD picture.

Acknowledgement We would like to thank D. Graudenz for many helpful discussions. We are grateful to the HERA machine group whose outstanding efforts made this experiment possible. We appreciate the immense effort of the engineers and technicians who constructed and maintain the $\mathrm{H} 1$ detector. We thank the funding agencies for financial support. We acknowledge the support of the DESY technical staff. We wish to thank the DESY directorate for the support and hospitality extended to the non-DESY members of the collaboration.

\section{References}

[1] D. Gross, F. Wilczek, Phys. Rev. Lett. 30, 1343 (1973).

[2] S. Bethke, J.E. Pilcher, Annual Review of Nuclear and Particle Science 42, 251 (1992). 
[3] JADE Collaboration, W. Bartel et al., Z. Phys. C33, 23 (1986).

[4] H1 Collaboration, I. Abt et al., Z. Phys. C61, 59 (1994).

[5] ZEUS Collaboration, M. Derrick et al., Phys. Lett. B306, 158 (1993).

[6] E665 Collaboration, M.R. Adams et al., Phys. Rev. Lett. 69, 1026 (1992), and Phys. Rev. Lett. 72, 466 (1994).

[7] D. Graudenz, Phys. Lett. B256, 518 (1991).

[8] D. Graudenz, Berkeley preprint, LBL-34147 (1993), published in Phys. Rev. D49, 3291 (1994).

[9] D. Graudenz, PROJET4.1, CERN-TH.7420/94.

[10] T. Brodkorb, J.G. Körner, Z. Phys. C54, 519 (1992);

T. Brodkorb, E. Mirkes, Univ. of Wisconsin, MAD/PH/820 (1994).

[11] B.R. Webber, Univ. of Cambridge, Cavendish-HEP-94/15.

[12] J.G. Körner, E. Mirkes, G.A. Schuler, Int. J. Mod. Phys. A4, 1781 (1989).

[13] H1 Collaboration, I. Abt et al., DESY 93-103.

[14] H1 Calorimeter Group, B. Andrieu et al., Nucl. Instr. Meth. A336, 460 (1993).

[15] H1 Calorimeter Group, B. Andrieu et al., Nucl. Instr. Meth. A350, 57 (1994).

[16] H1 Calorimeter Group, B. Andrieu et al., Nucl. Instr. Meth. A336, 499 (1993).

[17] S. Bentvelsen et al., Proc. Workshop 'Physics at HERA', DESY, Hamburg, Vol. 1, 23 (1991); K.C. Hoeger, ibid., Vol. 1, 43.

[18] H1 Collaboration, T. Ahmed et al., DESY 94-133;

ZEUS Collaboration, M. Derrick et al., Phys. Lett. B315, 481 (1993), and DESY 94-063.

[19] G. Ingelman, Proc. Workshop 'Physics at HERA', Hamburg, Vol 3, 1366 (1991).

[20] T. Sjoestrand, M. Bengtsson, Comput. Phys. Commun. 43, 367 (1987).

[21] W. de Boer, CERN-PPE-90-161.

[22] A.D. Martin, W.J. Stirling, R.G. Roberts, Phys. Rev. D47, 867 (1993).

[23] L. Lönnblad, ARIADNE version 4.03, Comput. Phys. Commun. 71, 15 (1992) and references therein.

[24] A.D. Martin, W.J. Stirling, R.G. Roberts, Durham, DTP 93-86, and Rutherford, RAL 93-077 (1993).

[25] H1 Collaboration, I. Abt et al., Nucl. Phys. B407, 515 (1993);

ZEUS Collaboration, M. Derrick et al., Phys. Lett. B316, 413 (1993).

[26] W. Bernreuther, W. Wetzel, Nucl. Phys B197, 228 (1982);

W.J. Marciano, Phys. Rev. D29, 580 (1984).

[27] R. Nisius, thesis, Aachen, PITHA 94/21 (1994).

[28] CTEQ Collaboration, H.L. Lai et al., Michigan State Univ., MSU-HEP-41024, (1994). 
[29] M. Glück, E. Reya, A. Vogt, Z. Phys. C53, 127 (1992).

[30] NMC Collaboration, M. Arneodo et al., Phys. Lett. B309, 222 (1993).

[31] e.g. S.J. Brodsky, G.P. Lepage, P.B. Mackenzie, Phys. Rev. D28, 228 (1983).

[32] Particle Data Group, L. Montanet et al., Phys. Rev. D50, 1297 (1994). 